\title{
Hospital antibiotic abuse and analysis of its adverse reactions
}

\author{
Yong Zhang* and Bin Guo
}

First Affiliated Hospital of Liaoning Medical University, Jinzhou, 121001, China.

Accepted 9 July, 2012

\begin{abstract}
The aim of this study was to analyze the use of antimicrobial drugs and assess the rationality of use of antimicrobial drugs. 433 random samples of hospital pharmacy prescription in June to August 2010 were selected, the prescriptions covered hospital representative subjects, the application of impatient antimicrobial drugs was described according to "Clinical Guide to Chinese Pharmacopoeia", "New Pharmacology", and the physicians' prescriptive habits and the recommended dose of new drug instructions. The results have shown that among the selected 433 cases, the irrational ratio of utilization of antibiotics was 245 cases, accounting for $56.6 \%$ of the investigated prescription. Underuse, due to insufficient access to effective antibiotics, inadequate dosing and poor adherence to therapy, may play as important a role in driving resistance as overuse. This widespread irrational use of drugs is one of the most important reasons for antibiotics losing their effectiveness. The findings suggest that doctors should be cautious in using antimicrobial drugs, selecting the appropriate dosage forms and paying attention to the rational use of drugs so as to reduce the incidence rate of adverse reactions so as to regulate the application of antibacterial drugs.
\end{abstract}

Key words: Inpatient prescription, antimicrobial drugs, irrational, prescription analysis.

\section{INTRODUCTION}

Currently, the antimicrobial drugs used clinically are so much and there have been no new antibiotics developed in the last 30 to 40 years, although there are organizations just focusing in this area of research. However, the lowest rate of use of antibiotics in China is up to $30 \%$; in rural areas, the data would become considerably acute (Xueju, 2012; Shi-ying et al., 2007). Thus, behind the harsh reality, we always choose the antibiotic when confronted with nearly every illness, even if the illness did not need the antibiotics. This paper analyzed the data of drug abuse of antibacterial drugs in order to provide basis for the rational use of antibiotics. It is important to recognize that a physician's ability to prescribe correctly can be compromised by a lack of laboratory diagnostic services or by poor laboratory performance; and failure to use the available diagnostic tests also promotes inappropriate use. Self-medication occurs regularly and has been recognized as one of the

*Corresponding author. E-mail: zhangyong287299563@163.com contributing factors for inappropriate use (Grigoryan et al., 2010). Thus, we should set up organizations aiming to improve prescribing of antibiotics in China by targeting both prescribers and patients (World Health Organization, 2011). Only when we act according to the ideas and the suggestions of the ReAct organization, we can get a healthy surviving environment in near future (ReAct, 2011).

\section{MATERIALS AND METHODS}

Sources

Statistics are the random samples of inpatient of First Affiliated Hospital of Liaoning Medical University from May to July, 2011; analysis was made according to the 433 selected prescriptions from five medical departments.

\section{Statistical methods}

Retrospective investigation was used to collect the information (gender, age, admission, discharge time, diagnosis, allergy history 
Table 1. Percentage of utilization and irrational utilization of Ab. in five medical departments.

\begin{tabular}{lcc}
\hline Medical department name & $\begin{array}{c}\text { No. of encounters } \\
\text { surveyed/day (average) }\end{array}$ & $\begin{array}{c}\text { No. of patients encountered with an } \\
\text { Ab. prescription (percentage) }\end{array}$ \\
\hline First Affiliated Hospital of Liaoning Medical University & $\begin{array}{c}\text { No. of encounters irrational } \\
\text { utilization of Ab. (percentage) }\end{array}$ \\
Department of Respiratory Medicine & 56 & $52(92.86)$ \\
Department of Neurology & 45 & $14(31.25)$ \\
General Surgery & 121 & $109(90.12)$ \\
Orthopedic Surgery & $135(48.22)$ & $123(91.43)$ \\
Thoracic Surgery & 76 & $63(83.81)$ \\
\hline
\end{tabular}

history, divisions and medication purpose) of the patients, medication (dosage, frequency/day, the total volume starting and ending time) and treatment outcomes. Determined daily dose (DDD) was determined according to the usual dose recommended by "Chinese Pharmacopoeia" and "New Pharmacology Methods". Drugs which were not incorporated had been determined by the legal drug specification. Drug use frequency (DUFs) $=$ Drug use $/ \mathrm{DDD}$, Drug use index $(\mathrm{DUI})=\mathrm{DUF} /$ the real time of taking drugs (Jing-su et al., 2007) were determined. The evaluation standard of assessing the rationality of taking drugs is DUI $<1$.

\section{RESULTS AND DISCUSSION}

In the past years, the use of antimicrobial drugs had been occupying an important position in the hospital medication. However, frequent use of antimicrobial drugs does not only increase the burden on the patient's bill, but also brought a lot of adverse reactions due to the abuse of antimicrobial drugs. Although antimicrobial drugs had been rapidly developed, but the incidence of hospital infectious diseases not only did not have a slight decline, but also shown a gradual upward trend. The hospital infection and infection therapy caused by inappropriate application of antimicrobial drugs had become one of the main tasks of the clinics. In this case, a reasonable application of antimicrobial drugs had become a primary task of the hospital clinical pharmacists.

\section{Different departments of antimicrobial drug use}

As shown in Table 1, all of the five medical departments had high rates of an irrational utilization of antibiotic drugs. However, antibiotic drugs were used in the general surgery, orthopedic surgery and thoracic surgery in the selected medical departments more frequently and were more likely to produce cases of irrational utilization of the antibiotic drugs than the other two departments.

\section{Combined medication of antibiotics and the irrational use of antimicrobial drugs}

As shown in Table 2, we can see that single drug prescription takes a large part of the whole prescription; it may be because only through this we can cut down the ratio of cases of adverse reactions. The ratio would be higher as the numbers of drug go up.

This study analyzes the reasons for the irrational use of antimicrobial drugs as shown in Table 4.

\section{Drug overdose or inefficiency, and} inappropriate course of treatment

Dose and drug effect should be noted with the application of anti-infective drugs. Overdoses will waste the drugs and increase the toxicity of drugs; inefficient doses will lead to generate bacterial resistance. To obtain a satisfactory therapeutic result, the concentration of antibacterial drugs in blood should be 2 to 10 times higher than the minimum inhibitory concentration (MIC), preferably 5 times more than the MIC (Duan, 2008). For the treatment programs, as soon as the obvious effect is observed, immediate withdrawal or non-withdrawal after the infection had been controlled for several days is likely to cause bacterial resistance. Antimicrobial drugs therapy will be different by levels of infection; in general, it was appropriate to act it out until body temperature is at $37^{\circ} \mathrm{C}$, namely 72 to $96 \mathrm{~h}$ after symptoms disappeared (Jin-you, 2009). Sepsis, infective endocarditis, typhoid fever, myelitis, hemolytic streptococcus pharyngitis and tonsillitis, deep mycosis, tuberculosis, etc. need longer 
Table 2. Antibiotics prescribed as a percentage share (proportionate distribution) of total antibiotics prescribed per day.

\begin{tabular}{|c|c|c|c|c|c|}
\hline Antibiotic category & $\begin{array}{l}\text { Percentage share of Ab. in } \\
\text { Department of Respiratory Medicine }\end{array}$ & $\begin{array}{l}\text { Percentage share of Ab. in } \\
\text { Department of Neurology }\end{array}$ & $\begin{array}{c}\text { Percentage share of } A b . \text { in } \\
\text { General surgery }\end{array}$ & $\begin{array}{c}\text { Percentage share of Ab. in } \\
\text { Orthopedic Surgery }\end{array}$ & $\begin{array}{c}\text { Percentage share of } \mathbf{A b} . \text { In } \\
\text { Thoracic Surgery }\end{array}$ \\
\hline \multicolumn{6}{|l|}{ Penicillins } \\
\hline Amoxicillin & 32 & 21.1 & 62.3 & 64.6 & 45.2 \\
\hline \multicolumn{6}{|l|}{ Ampicillin } \\
\hline \multicolumn{6}{|l|}{ Quinolones } \\
\hline Norfloxacin & 10.2 & 11.3 & 25 & 23.4 & 12.7 \\
\hline \multicolumn{6}{|l|}{ Ciprofloxacin } \\
\hline \multicolumn{6}{|l|}{ Cephalosporins } \\
\hline \multicolumn{6}{|l|}{ Amoxicillin } \\
\hline Potassium clavulanate & 7.5 & 8.3 & 9.2 & 7 & 8.6 \\
\hline \multicolumn{6}{|l|}{ Cefadroxil } \\
\hline \multicolumn{6}{|l|}{ Ceftriaxone } \\
\hline \multicolumn{6}{|l|}{ Aminoglycosides } \\
\hline Streptomycin & 10.2 & 12 & 18.5 & 16.8 & 12.4 \\
\hline Kanamycin & & & & & \\
\hline
\end{tabular}

Table 3. Combined medication analysis.

\begin{tabular}{lcc}
\hline $\begin{array}{l}\text { Combined medication } \\
\text { program }\end{array}$ & $\begin{array}{c}\text { The number of combination } \\
\text { drugs prescription }\end{array}$ & $\begin{array}{c}\text { Total prescription ratio } \\
(\%)\end{array}$ \\
\hline Single-drug & 175 & 71.68 \\
Two-drug & 64 & 26.13 \\
Three-drug & 5 & 2.19 \\
\hline
\end{tabular}

courses of treatments to prevent and cure reoccurrence (Chang-qin, 2003).

\section{Irrational dosage regimen}

As shown in Table 3, the main types of irrational use of antimicrobial drugs include the irrational regimen and non-indications medication. Choosing the wrong drug will have a big impact on the treatment of infectious diseases. Thus, the utilization of drug should be optimized according to the antibacterial test results and levels of infection.

Another important issue is that the degree of attention of doctors on the antibiotics' potential side effects was not enough, and they prescribe only by virtue of their own experience to prescribe mechanically.

\section{No indications antibiotics}

Non-indication antimicrobial drug is typical performance of irrational drug use. Such prescriptions were accounted for $25.50 \%$ of the irrational prescription, mainly for anti-infective treatment of non-infection indications (Nian-hui, 2002). Such as one male patient was diagnosed with appendicitis, the prescription was as follows: 
Table 4. Analysis of the reasons for the irrational use of antimicrobial drugs.

\begin{tabular}{lccc}
\hline Type & Number of cases & Irrational prescription ratio (\%) & Total prescription ratio (\%) \\
\hline Irrational drug selection program & 57 & 23.28 & 13.16 \\
Irrational dose treatment & 4 & 10.78 & 0.92 \\
No indications of drug use & 62 & 25.70 & 14.3 \\
Irrational combined medication & 99 & 40.44 & 22.86 \\
\hline
\end{tabular}

Table 5. Rationality evaluation of medication through drug DUI size.

\begin{tabular}{lccc}
\hline Drug name & Use cases times & DUFS & DUI \\
\hline Amoxicillin & 64 & 656 & 1.56 \\
Ampicillin & 50 & 142 & 1.12 \\
Norfloxacin & 40 & 73 & 0.52 \\
Ciprofloxacin & 37 & 143 & 1.52 \\
Amoxicillin & 32 & 256 & 0.96 \\
Potassium clavulanate & 28 & 132 & 1.04 \\
Cefadroxil & 26 & 114 & 0.72 \\
Ceftriaxone & 20 & 130 & 0.86 \\
Streptomycin & 43 & 164 & 1.73 \\
\hline
\end{tabular}

$0.75 \mathrm{mg}$ ampicillin sulbactam injection sterile powder plus $5 \%$ glucose injection $100 \mathrm{ml}$, intravenous infusion; in addition, diarrhea in children is mainly because rotavirus infection, appropriate rehydration and probiotics can significantly shorten the duration of diarrhea of children, there is no need to use the antimicrobial drugs (Guo-ying, 2007). Another example was that one case of lumbar sprain found in a female patient; the clinic gave the antiinfective pefloxacin injection sterile powder $0.4 \mathrm{mg}+5 \%$ glucose injection $250 \mathrm{ml}$, intravenously, and so on. These two cases found that no indications of anti-infection treatment were an excellent reason for high rate of bacterial resistance (Shui-qu, 2003).

\section{Irrational combined application and evaluation of the rationality of medication}

As shown in Table 2, 69 cases were treated with drugs combination medication, in which only 183 cases have clear indications, the remaining majorities are infections or prophylaxis. This application of the antimicrobial drugs should only be adapted to the clear symptom, inappropriate combined application would not get the desired effect and would increase the health burden and adverse reactions; therefore, serious infection will come out (Riechelmann, 2007). In respiratory infections, intravenous infusion of cefotaxime sodium and ornidazole; intravenous infusion of amoxicillin and ornidazole; intravenous infusion of azithromycin and levofloxacin; for upper respiratory tract infection, most of which are viral infections, do not need combined medication. This will not only increase the economic burden of patients, but also easily cause the antimicrobial drug resistance (Classen, 2007). So the doctors should be careful at the choice of combined medication and control the indication to improve the diagnostic quality and make the combined application of antibacterial drugs really cure the severe mixed infection / expansion of the antibacterial spectrum, to reduce the incidence of drug resistance and reduce the role of adverse drug reactions. Rationality of the medication was evaluated through drug DUI size analysis as shown in Table 5.

\section{Use frequency order of antimicrobial drugs}

As shown in Table 4, the most frequently used drugs in the hospital were the penicillins, DUI $>1$. The other similar drugs were cephalosporins, aminoglycosides and quinolones. The evaluation standard of assessing the rationality of taking drugs is $\mathrm{DUI}<1$. In the hospital, the dosage of these drugs was higher than 1. Re-inspecting the prescription, we found that doctors issued azithromycin, a one-time issuance of drug use generally cannot be longer than 7 days, the amount of the provisions of the "prescription management approach"; Ornidazole tablets usage was generally $500 \mathrm{mg} / \mathrm{times}, 2$ times/day, while in the prescription, it was $500 \mathrm{mg} / 3$ times/day" usage (Sarayaa, 2004), which may result in the DUI exception of drugs, indicating that the hospital antimicrobial drug use was basically rational, but individual doctors are not yet good at grasping the usage and consumption of drugs, so it was necessary to strengthen 
the rational use of medical staff education (Bertino et al., 2002).

\section{Conclusions}

In recent years, anti-bacterial drug abuse has become serious, and in some primary health care centers, antimicrobial drugs were even regarded as a "panacea". Once antimicrobial drugs were abused, not only was it not conducive to health, but also causes serious damage to the human body. The irrational use of antimicrobial agents will lead to the drug resistance, toxicity and allergic reactions. Therefore, rational use of antibiotics is a serious problem faced by the clinician. It had been raised to arouse enough attention.

\section{ACKNOWLEDGEMENT}

This work was supported by First Affiliated Hospital of Liaoning Medical University.

\section{REFERENCES}

Bertino JS Jr, Owen RC Jr, Cames TD (2002). Gatifloxacin associated corrected Q-T interval prolongation, torsades depoints, and ventricular fibrillation in patients with known risk factors. Clin Infect Dis. 34(6): 861-863.

Chang-qin H (2003). Antimicrobial Drugs in Free between Abuse and Quality control. China Prescription Drug, 12(12): 21-22

Classen DC (2007). The timing of prophylactic administration of antibiotics and the risk of surgical wound infection. N Engl Med. 32(6):281.

Duan L (2008). Pharmacology [M]. 6th ed. Beijing: People's Health Publishing House. pp. 4-8.

Grigoryan L, Monnet DL, Haaijer-Ruskamp FM, Bonten MJ, Lundborg S, Verheij TJ (2010). Self-medication with antibiotics in Europe: A case for action. Curr. Drug Saf. 5(4):329-32.
Guo-ying C (2007). Analysis of Irrational Drug Use in Outpatient Prescription. J. Pharm. Practice. 25 (9):778.

Jing-su L, Chang-chun C, Yan-ning H (2007). Investigation and Analysis of Hospital Clinical Use of Antibiotics. Chinese Pharmacists, 10:10411043.

Jin-you Y (2009). Prescription Analysis of Irrational Drug Use. Cap. Med. 22:47-48.

Nian-hui X, Guo-sheng L, Jie F (2002). Discussion on Management Experience on Rational Use of Antibiotic Drugs Chinese J. Nosocomiolo. 12(2):143-144.

ReAct (2011). Action on Antibiotic Resistance. p. 3.

Riechelmann RP, Tannock IF, Wang L (2007). Potential drug interactions and duplicate prescriptions among cancer patients. J. Netl Cancer Inst. 99(8):592-560.

Sarayaa $Y$ (2004). Effects of fluoroquinol ones on insulin secreti on and $\beta$ cell ATP sensitive K+ channels. Eur. J. Pharm. acol. 49(7):112-115.

Shi-ying G, Yun Y, Zhong-liang D (2007). Bacterial Population Distribution in the environment of community outside and Resistance Testing. Chin. J. Health Laboratory Technolo. 17(10):1830-1837.

Shui-qu Liu (2003). Some Problems of the Application of Antibiotics. Zhejiang J. Clinical Med. 5(8):561-562.

World Health Organization (2011). The evolving threat of antimicrobial resistance Options for action. pp. 3-42.

Xueju Li (2012). Analysis of consequences of misuse of antibiotics. Chin. J. Clin. Rat. Drug Use 5:4B-130. 